\title{
Theoretical Study of the Effect of Light on Nematic to Isotropic Phase Transition
}

\author{
B.L. Suresha ${ }^{a}$, M.C. Radhakrishna ${ }^{a}$ And A.S. Govind ${ }^{b, *}$ \\ ${ }^{a}$ Dept. of Physics, Bangalore University, Bangalore, 560 056, India \\ ${ }^{b}$ Dept. of Physics, Vijaya Degree College, Bangalore, 560 004, India
}

(Received August 18, 2010; in final form April 20, 2011)

\begin{abstract}
Optical response of liquid crystals is very important from the point of view of applications of liquid crystals in display and data storage devices. Experiments have shown that UV rays incident on nematic liquid crystals doped with photoactive azo compounds lower the nematic isotropic transition temperature $\left(T_{\mathrm{NI}}\right)$. This is attributed to the transformation of photoactive trans isomer to cis isomer due to UV rays. We have earlier developed a mean field model involving molecular explanation for two lengths in which the mutual orientation of near neighbor molecules changes from an antiparallel to a parallel configuration as the temperature lowered. In this paper, this model is extended to include the length change from trans to cis isomer. The calculated values of change in $T_{\mathrm{NI}}$ qualitatively agree with experimental trends.
\end{abstract}

PACS: 61.30.-v, 64.70.M-

\section{Introduction}

Liquid crystals are fluids characterized by long-range orientational order without long-range three-dimensional translational molecular order $[1,2]$. They have some properties of liquids (for example, they can flow) as well as some of those of crystals (for example, birefringence). They consist of molecules having pronounced shape anisotropy (rod-like, disc-like or with a bent core). Liquid crystals obtained by heating the solid crystals are called thermotropic liquid crystals.

Mainly, there are two fundamentally different types of liquid crystals made of rod-like molecules namely, the nematic and the smectic.

The extent of orientational order of the uniaxial nematic liquid crystal is represented by the so-called scalar order parameter $S$ defined as [1]:

$$
S=\frac{1}{2}\left\langle 3 \cos ^{2} \theta_{i}-1\right\rangle,
$$

where $\theta_{i}$ is the angle between the long axis of the $i$-th molecule and the director. The order parameter takes a maximum value equal to 1 when all the rods are perfectly aligned and is zero when all the orientations of the long axes are equally probable (in the isotropic phase).

When a typical mesomorphic compound is cooled from the isotropic (I) phase, the usual sequence of phase transitions is (with $\mathrm{K}$ for solid crystal): I $\rightarrow \mathrm{N} \rightarrow \mathrm{K}$. The $T_{\mathrm{NI}}$ is the transition tempetature at which the nematic to isotropic transition occurs.

In recent years, a field of research that is growing steadily is the photoinduced phenomenon in which the

\footnotetext{
* corresponding author; e-mail: govindas63@gmail.com
}

incident light itself brings about molecular ordering/disordering of the liquid crystalline system [3]. Photonics, in which light can be controlled by light as a stimulus, is being proposed as the future technology for high-speed information processing [4]. It is well known that liquid crystals are convenient to control light because they exhibit large optical effects owing to reorientation of the molecules with the application of low-magnitude electric fields. It has been shown [5] that even in nonphotoabsorbing liquid crystals, optical fields, albeit large, can be used to cause a similar reorientation. However, an addition of chromophores, such as azo groups, has been found to lower the required optical field significantly [6]. Furthermore, the photoinduced birefringence has extremely high long-term stability and holograms recorded show hardly any degradation. This feature has made the photochromic materials, in particular the azo compounds, very promising candidates for optical data storage. An illustration of nature utilizing photoinduced effects is that of the biological photochrome bacterio-rhodopsin found in the eye. Apart from their biological and technological importance, these systems exhibit photodriven phase transitions, thus providing a new tool to study phase transitions and the associated critical phenomena. Several groups have done extensive work in this field [7].

Photoinduced effects reported in the literature are on liquid crystals in which the azo group is either chemically attached to the molecule or is used as a dopant in a liquid crystal host material. If the material exhibits a nematic-isotropic $(\mathrm{N}-\mathrm{I})$ transition and the UV irradiation is done in the nematic phase, the lowering of the transition temperature $\left(T_{\mathrm{NI}}\right)$ could induce an isothermal $\mathrm{N}-\mathrm{I}$ transition. It is this photochemically induced transition that is promising for the optical image-storing systems. One of the requirements for such an application 
is that the lowering of $T_{\mathrm{NI}}$ is appreciable [8]. Although, large shifts in $T_{\mathrm{NI}}$ can be obtained if the host material itself is photoactive [9]. From the operational point of view, it is obviously better to dope small quantities of guest photoactive material into a host liquid crystalline system which has been optimized for temperature range, viscosity, etc. Molecules containing azo groups are well known to show reversible isomerization transformations upon irradiation with UV and visible light.

One salient feature observed is the drastic decrease by $15^{\circ} \mathrm{C}$ in the value of $T_{\mathrm{NI}}$ on UV exposure. $\Delta T\left[=T_{\mathrm{NI}}\right.$ (without UV) $-T_{\mathrm{NI}}$ (with UV)] is also affected by pressure [10]. The experimental results are as shown in Fig. 1.

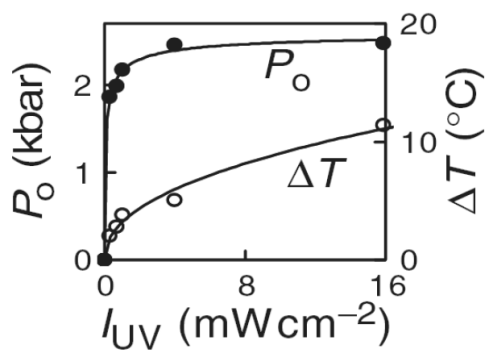

Fig. 1. The intensity dependence of $\Delta T$ and that of the pressure at which $\Delta T$ goes to zero for a given intensity [10].

Molecular interpretation of this is as follows. Upon UV irradiation around $365 \mathrm{~nm}$, the energetically more stable trans configuration, with an elongated rod-like molecular form changes into a bent or cis configuration (Fig. 2). The reverse transformation can be brought about by illuminating with visible light (in the range $400-500 \mathrm{~nm}$ ).

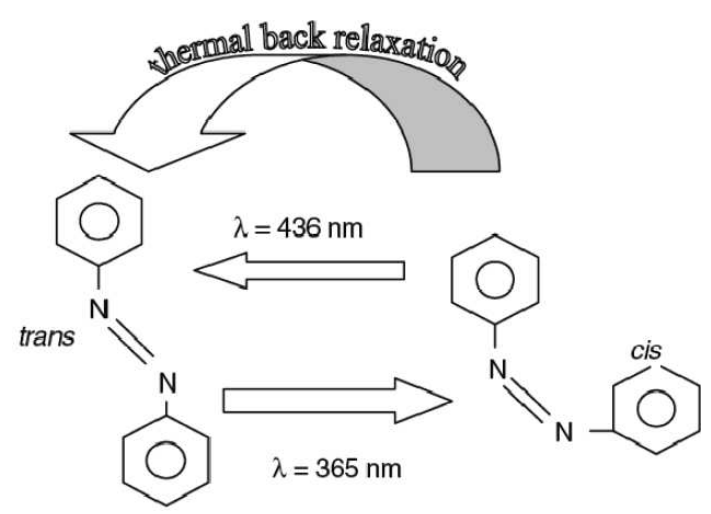

Fig. 2. Schematic diagram illustrating the trans-cis and cis-trans isomerizations [10].

The latter change also occurs in the "dark" by a process known as "thermal back relaxation" in a period ranging from minutes to tens of hours depending on the system [11]. In the case of the photoactive guest-non-photoactive host systems, the trans form of the azo dopant, as it is rod-like, is favourable for the stabilization of the liquid-crystalline phase. On the other hand, the cis form, which is in bent form, acts like an "impurity" and therefore destabilizes the liquid-crystalline phase. Hence, photoisomerization from the trans to the cis form causes, in general, a lowering of the transition temperature.

\section{Molecular model}

The host medium has molecules with polar cyano end group. Such a medium has intrinsic ability to show re-entrant phenomena. This is explained considering the medium to be consisting of "two lengths". The trans to cis change of the guest molecules in the medium also results in a system consisting of "two lengths". Thus, the medium is assumed to have "two lengths", with the fraction of shorter molecules varying with irradiation of UV and also with temperature.

A simple model to explain the molecular origin of the "two lengths" assumed in the Prost phenomenological model was proposed by Madhusudana and Jyothsna Rajan [12]. The basic concept in this model is that the molecular pairs can change over from antiparallel (A) to parallel $(\mathrm{P})$ configuration as the intermolecular separation $(r)$ is reduced due to cooling or due to increase of pressure. The medium is treated as an equilibrium mixture of the A and $\mathrm{P}$ types of pairs. Experiments [13] showing the presence of polar short range order at low temperatures support this model. In the model proposed by Madhusudana and Jyothsna Rajan [12], the origin of the two incommensurate lengths is explained as follows: the permanent dipolar interaction favours an antiparallel arrangement between the neighbouring mesogenic molecules [14]. However, the aromatic part of the antiparallel neighbours overlap due to the strong dispersion interaction between them leading to the partial bilayer arrangement (see Fig. 3a). In this configuration, the alkyl chains of the two molecules, which lie on opposite sides of the core region, do not have a significant interaction. On the other hand, if the molecules are parallel, the permanent dipolar interaction is repulsive. However, the aromatic cores have strong polarizabilities and the induced dipole moment due to a neighbouring polar molecule would weaken the net dipole moment of any given molecule in this configuration (see Fig. 3b).

Further, the chains of the two neighbours are now in close proximity and the dispersion interaction between them would favour this arrangement. The repulsive dipolar interaction is $\propto 1 / r^{3}$ where $r$ is the intermolecular separation, while both the dipole-induced dipole and the dispersion interactions are $\propto 1 / r^{6}$ and are attractive in nature. Hence there can be a change from the antiparallel to the parallel configuration as the intermolecular separation is decreased below some value as the density is increased due to a lowering of temperature or an increase of pressure.

The calculated values [15] of $\Delta E=E_{\mathrm{A}}-E_{\mathrm{P}}$ for various intermolecular separations are shown in Fig. 4. This clearly shows favouring of $\mathrm{P}$ type at lower intermolecular separations. 


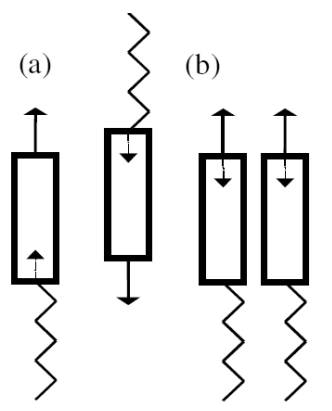

Fig. 3. Schematic diagram showing (a) the antiparallel configuration of two molecules favoured at intermediate molecular separations and (b) the parallel configuration favoured at relatively low values of intermolecular separation. The arrow with solid line represents the permanent dipole moment and the one with dotted line, the induced one. (For the sake of clarity, the relative separation in (a) is exaggerated.).

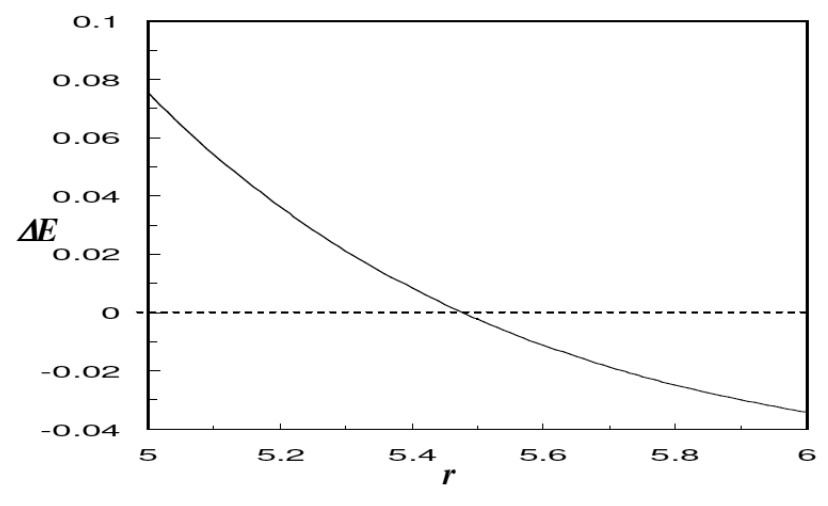

Fig. 4. The pairing energy difference $\Delta E=E_{\mathrm{A}}-E_{\mathrm{P}}$ (in $10^{-19} \mathrm{~J}$ ) between the antiparallel and parallel configurations plotted as a function of the intermolecular separation $r$ (in $\AA$ ).

This concept has already been used by Govind and Madhusudana to explain $\mathrm{N}_{\mathrm{l}}-\mathrm{N}_{\mathrm{d}}$ transition, double and quadruple re-entrance, effect of electric field etc. [16].

We extend this molecular theory [17] of highly polar compounds to include the effect of light. Many of the assumptions made in this model have already been discussed in the earlier papers [16]. We recall the assumptions relevant for the present theory.

\subsection{Assumptions}

As explained above, the medium is assumed to consist of "pairs" of molecules having either antiparallel (A) or parallel $(\mathrm{P})$ configurations. The difference between the pairing energy of the A-type $\left(E_{\mathrm{A}}\right)$ and the P-type $\left(E_{\mathrm{P}}\right)$ configurations is written as

$$
\Delta E=E_{\mathrm{A}}-E_{\mathrm{P}}=R_{1} k_{\mathrm{B}} T_{\mathrm{NI}},
$$

where $R_{1}$ is an interaction parameter and $R_{2}$ is the reduced temperature at which the density of the medium is such that $\Delta E$ becomes zero and $T_{R}=T / T_{\mathrm{NI}}$.
The orientational potential for A-type of pairs $\left(U_{\mathrm{AA}}\right)$ and P-type of pairs $\left(U_{\mathrm{PP}}\right)$ are assumed to be different

$$
U_{\mathrm{PP}}=Y U_{\mathrm{AA}}
$$

and the mutual interaction potential

$$
U_{\mathrm{AP}}=U_{\mathrm{PA}}=P \sqrt{U_{\mathrm{AA}} U_{\mathrm{PP}}},
$$

where $P \neq 1$ indicates a deviation from the geometric mean (GM) approximation.

The following are the additional assumptions made to incorporate the effect of light.

As explained in Sect. 1, the molecular configuration changes from cis to trans upon irradiation. Their respective lengths [18] are about $2.41 \mathrm{~nm}$ and $1.5 \mathrm{~nm}$. Thus, in our model, the A-type pairs are of the size of cis molecules and the P-type pairs match with the trans molecules. For simplicity in calculations we consider pairs of cis and trans molecules in the equations. Hence, irradiation of UV increases the number of molecular pairs of cis type, which is equivalent to increase of mole fraction of P-type pairs $\left(X_{\mathrm{P}}\right)$. The same effect would have occurred upon increase of $E_{\mathrm{P}}$. An increase of $E_{\mathrm{P}}$ results in the decrease of $\Delta E$. This is equivalent to shifting the graph downwards in Fig. 4. In other words, effectively $R_{2}$ decreases. Thus, $R_{2}$ with light $\left(R_{2}(L)\right)$ is less than $R_{2}$ without light $\left(R_{2}(0)\right)$.

In view of this, we can write

$$
R_{2}(L)=R_{2}(0)-\delta,
$$

where $\delta$ represents the change in $R_{2}$ because of light.

We calculate $\delta$ as follows:

Let $\mathrm{d} E$ be the amount of light energy incident on the sample in a time $\mathrm{d} t$. Due to this, some trans molecules change to cis. Let the number of trans (i.e., A type) molecules change from $N_{\mathrm{A}}$ to $N_{\mathrm{A}}-\mathrm{d} N_{\mathrm{A}}$.

Thus,

$$
\begin{aligned}
& -\frac{\mathrm{d} N_{\mathrm{A}}}{N_{\mathrm{A}}} \propto \mathrm{d} E, \\
& -\frac{\mathrm{d} N_{\mathrm{A}}}{N_{\mathrm{A}}}=K_{1} \mathrm{~d} E,
\end{aligned}
$$

where $K_{1}$ is a constant to be estimated later.

As mentioned earlier, the reverse transformation of the cis isomer into the trans isomer (thermal back relaxation) takes a very long time. Thus, the fraction of cis changing back to trans is negligibly small. Hence, this change during UV irradiation is ignored.

Solving this, we get,

$$
N_{\mathrm{A}}=N_{\mathrm{A}}(0) \mathrm{e}^{-K_{1} E},
$$

where $N_{\mathrm{A}}(0)$ is the number of trans pairs in the absence of light.

The number of cis (i.e., $\mathrm{P}$ type) molecules resulting is

$$
N_{\mathrm{P}}=N_{\mathrm{A}}(0)-N_{\mathrm{A}}=N_{\mathrm{A}}(0)\left[1-\mathrm{e}^{-K_{1} E}\right] \text {. }
$$

We assume that the change in $R_{2} \propto N_{\mathrm{P}}$. Thus, $\delta=$ $K_{2}\left(1-\mathrm{e}^{-K_{2} E}\right)$, or

$$
R_{2}=R_{2}(0)-K_{2}\left(1-\mathrm{e}^{-K_{1} E}\right) .
$$


Thus,

$$
\frac{\Delta E}{k T}=R_{1}\left[\frac{R_{2}(0) K_{2}\left(1-\mathrm{e}^{\mathrm{K}_{1} \mathrm{E}}\right)}{T_{R}}-1\right] .
$$

The values of $K_{1}$ and $K_{2}$ are estimated as explained later in Sect. 2.3.

\subsection{Free energy and order parameters}

Extending the Maier-Saupe theory for mixtures, the potential energy of the $i$-th A-type of pair can be written as

$$
\begin{gathered}
U_{\mathrm{A} i}=-U_{\mathrm{AA}} X_{\mathrm{A}} S_{\mathrm{A}} P_{2}\left(\cos \theta_{\mathrm{A} i}\right) \\
-U_{\mathrm{AP}} X_{\mathrm{P}} S_{\mathrm{P}} P_{2}\left(\cos \theta_{\mathrm{A} i}\right),
\end{gathered}
$$

where $X_{\mathrm{A}}, X_{\mathrm{P}}, S_{\mathrm{A}}, S_{\mathrm{P}}$ are the mole fractions, orientational of $\mathrm{A}$ and $\mathrm{P}$ types of pairs, respectively. Similarly for a P-type pair, $U_{\mathrm{P} j}$ is obtained by interchanging suffixes $\mathrm{A}$ and $\mathrm{P}$ in Eq. (6). The internal energy of one mole of pairs can be written as

$$
2 U=\frac{N X_{\mathrm{A}}}{2}\left\langle U_{\mathrm{A} i}\right\rangle+\frac{N X_{\mathrm{P}}}{2}\left\langle U_{\mathrm{P} j}\right\rangle-N X_{\mathrm{P}} \Delta E,
$$

where the angular brackets indicate statistical average, the factor 2 on the left hand side reminds that we have a mole of pairs, the factor $1 / 2$ appears in the first two terms since each pair is counted twice while averaging over the mutual interactions and we have also added the concentration dependent part of the configurational energy. The molar entropy is given by

$$
\begin{aligned}
2 \zeta & =-N k_{\mathrm{B}}\left[X_{\mathrm{A}} \frac{1}{d} \int_{-d / 2}^{+d / 2} \mathrm{~d} z_{\mathrm{A} i} \int_{0}^{1} \mathrm{~d}\left(\cos \theta_{\mathrm{A} i}\right) f_{\mathrm{A} i} \ln f_{\mathrm{A} i}\right. \\
& \left.+X_{\mathrm{P}} \frac{1}{d} \int_{-d / 2}^{+d / 2} \mathrm{~d} z_{\mathrm{P} j} \int_{0}^{1} \mathrm{~d}\left(\cos \theta_{\mathrm{P} j}\right) f_{\mathrm{P} j} \ln f_{\mathrm{P} j}\right] \\
& -N k_{\mathrm{B}}\left(X_{\mathrm{A}} \ln X_{\mathrm{A}}+X_{\mathrm{P}} \ln X_{\mathrm{P}}\right),
\end{aligned}
$$

where the last term is the entropy of mixing and $f_{\mathrm{A}}$ and $f_{\mathrm{P}}$ are the normalised distribution functions of $\mathrm{A}$ and $\mathrm{P}$ types of pairs, respectively. The Helmholtz free energy is given by

$$
F=U-T \zeta .
$$

The orientational distribution functions of the A-type of pairs $f_{\mathrm{A}}$ and $f_{\mathrm{P}}$ are found by minimising $F$.

We have

$$
\begin{aligned}
f_{\mathrm{A}} & =\frac{1}{Z_{\mathrm{A}}} \exp \left(\left(\frac{U_{\mathrm{AA}}}{k_{\mathrm{B}} T}\left[X_{\mathrm{A}} S_{\mathrm{A}}+P \sqrt{Y} X_{\mathrm{P}} S_{\mathrm{P}}\right]\right)\right. \\
& \left.\times P_{2}\left(\cos \theta_{\mathrm{A}}\right)\right), \\
f_{\mathrm{P}} & =\frac{1}{Z_{\mathrm{P}}} \exp \left(\left[\frac{U_{\mathrm{AA}}}{k_{\mathrm{B}} T}\left(Y X_{\mathrm{P}} S_{\mathrm{P}}+P \sqrt{Y} X_{\mathrm{A}} S_{\mathrm{A}}\right)\right]\right. \\
& \left.\times P_{2}\left(\cos \theta_{\mathrm{P}}\right)\right),
\end{aligned}
$$

where $Z_{\mathrm{A}}$ and $Z_{\mathrm{P}}$ are the appropriate normalising integrals. Hence the order parameters are given by

$$
S_{\mathrm{A}}=\int_{0}^{1} \mathrm{~d}\left(\cos \theta_{\mathrm{A} i}\right) P_{2}\left(\cos \theta_{\mathrm{A} i}\right) f_{\mathrm{A}},
$$

where $S_{\mathrm{P}}$ is obtained by interchanging the suffixes A and $\mathrm{P}$ in Eq. (7). The free energy per mole of pairs can now be written in the simplified form

$$
\begin{aligned}
2 F & =+\frac{N U_{\mathrm{AA}}}{2}\left[X_{\mathrm{A}}^{2} S_{\mathrm{A}}^{2}+Y X_{\mathrm{P}}^{2} S_{\mathrm{P}}^{2}\right. \\
& \left.+2 P \sqrt{Y} X_{\mathrm{A}} X_{\mathrm{P}} S_{\mathrm{A}} S_{\mathrm{P}}\right]-N k_{\mathrm{B}} T X_{\mathrm{A}} \ln \left(\frac{Z_{\mathrm{A}}}{X_{\mathrm{A}}}\right) \\
& -N k_{\mathrm{B}} T X_{\mathrm{P}} \ln \left(\frac{Z_{\mathrm{P}}}{X_{\mathrm{P}}}\right)-N X_{\mathrm{P}} \Delta E .
\end{aligned}
$$

The equilibrium value of the mole fraction of the A-type of pairs $\left(X_{\mathrm{A}}\right)$ is found by minimising $F$ with respect to $X_{\mathrm{A}}$. We get, with $X_{\mathrm{P}}=1-X_{\mathrm{A}}$,

$$
\frac{X_{\mathrm{P}}}{X_{\mathrm{A}}}=\frac{Z_{\mathrm{P}}}{Z_{\mathrm{A}}} \exp \left(\frac{\Delta E}{k_{\mathrm{B}} T}\right) .
$$

\subsection{Calculations}

Calculations have been made as for $R_{2}(0)=0.85$ with $R_{1}=10, P=0.6$ and $Y=0.8$ which are reasonable as explained in Ref. [19]. We evaluate all the necessary integrals using a 32 point Gaussian quadrature method in double precision. We look for the following types of solutions:

1. $S_{\mathrm{A}}, S_{\mathrm{P}} \neq 0$ leading to nematic phase and

2. $S_{\mathrm{A}}, S_{\mathrm{P}}=0$ leading to isotropic phase.

The stable phase is the one having lower free energy. The temperature at which these two solutions have the same free energy gives $T_{\mathrm{NI}}$.

The experiments show that trans to cis changing efficiency is quite high over a large temperature range, with the maximum occurring in the vicinity of the $T_{\mathrm{NI}}$ of the irradiated case. In fact [10] the efficiency remains within $20 \%$ of the maximum over a range of $10^{\circ} \mathrm{C}$.

The change in $\Delta E$ due to light corresponds to the energy difference between trans and cis configurations. This corresponds to the energy difference between $365 \mathrm{~nm}$ and $435 \mathrm{~nm}$. This is about $22 k_{\mathrm{B}} T$ at $T=300 \mathrm{~K}$. However, all the light energy incident is not used for trans to cis conversion because the photo absorbing material is doped to about $5 \%$ and the maximum photo efficiency is about $20 \%$. Thus average energy per molecule reduces to $(1 / 100)$-th of $22 k_{\mathrm{B}} T$, i.e., $0.22 k_{\mathrm{B}} T$. For a pair of molecules, it is $0.44 k_{\mathrm{B}} T$. Hence, we have used $0.5 k_{\mathrm{B}} T$.

Thus, the change in $\Delta E$ due to light is

$$
\frac{\delta(\Delta E)}{k_{\mathrm{B}} T}=0.5 \text {. }
$$

In Eq. (5), the change in $\Delta E$ due to change in $R_{2}$ is

$$
\frac{\delta(\Delta E)}{k_{\mathrm{B}} T}=\frac{R_{1} K_{2}\left(1-\mathrm{e}^{-K_{1} E}\right)}{T_{R}} .
$$


Here, $K_{2}$ represents the maximum change in $R_{2}$ for light energy $E \rightarrow \infty$. This is assumed to be 0.1. We are investigating the change in $T_{\mathrm{NI}}$ due to light. Thus, our calculations are for $T_{R}$ close to 1 . For $R_{1}=10$ and $T_{R}=1$, we have

$$
\begin{aligned}
& K_{2}\left(1-\mathrm{e}^{-K_{1} E}\right)=\frac{0.5 T_{R}}{R_{1}}=0.05, \\
& \mathrm{e}^{-K_{1} E}=1-\frac{0.05}{0.1}=0.5, \\
& K_{1} E=\ln 2=0.7 .
\end{aligned}
$$

For $E=1 \mathrm{~mJ} \mathrm{~cm}{ }^{-2}$ and the magnitude of $K_{1}=0.7$ with $E$ in $\mathrm{mJ} \mathrm{cm}{ }^{-2}$, Eq. (3) becomes

$$
R_{2}(L)=R_{2}(0)-0.1\left(1-\mathrm{e}^{-0.7 E}\right) \text {. }
$$

\section{Results and discussions}

The incidence of UV rays change the trans isomer (longer) to cis isomer (shorter). This is equivalent to an increase in shorter "P" type of pairs. The "P" type pairs which are shorter have lower orientational potential than "A" type pairs. Thus we have used $Y=0.8$ in Eq. (2), as in earlier papers [19]. Thus, the medium with larger $X_{\mathrm{P}}$ has lower $T_{\mathrm{NI}}$. Hence, UV irradiation results in lowering of $T_{\mathrm{NI}}$. This is indirectly brought about by variation of $R_{2}$. As mentioned earlier, the thermal back relaxation is very slow and the relaxation of cis to trans and hence the decrease of $X_{\mathrm{P}}$ is ignored. As the intensity of UV radiation is increased, the fraction of trans available reduces. Thus, the variation of $\Delta T_{\mathrm{NI}}$ with intensity is not linear. The slope decreases indicating that the cis isomers are gradually reaching saturation. The total energy received also depends on the time of illumination. Thus we have plotted $\Delta T_{\mathrm{NI}}$ with respect to energy not power.

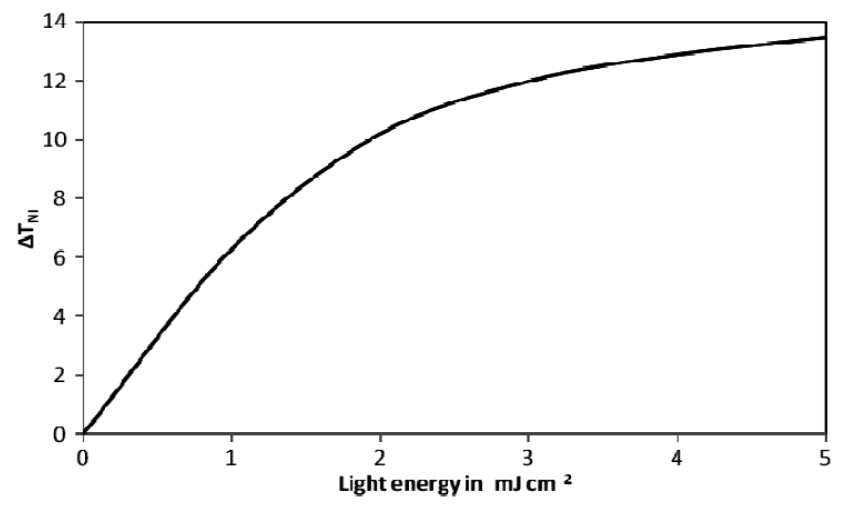

Fig. 5. Variation of calculated values of $\Delta T_{\mathrm{NI}}$ in $\mathrm{K}$ as a function of incident light energy in $\mathrm{mJ} \mathrm{cm}^{-2}$.

Assuming the time duration of illumination to be same, it can be compared with the experimental graph of $\Delta T_{\mathrm{NI}}$ with respect to intensity shown in Fig. 1. The trends agree qualitatively.

\section{Conclusions}

Response of liquid crystals to light is very important for applications of liquid crystals in display and memory devices. Recently experiments have been carried out on liquid crystals doped with photoactive azo compounds. It is seen that UV rays incident on such systems can lower the nematic isotropic transition temperature $T_{\mathrm{NI}}$. This is attributed to the change in trans (longer) isomer to cis (shorter) isomer of photoactive dopant. We have earlier developed a molecular mean field model assuming the medium to consist of inter-converting antiparallel and parallel pairs to explain the origin of "two lengths". The model was used to explain double re-entrance, effect of electric field on $T_{\mathrm{NI}}$ etc. This model is modified to include the change of trans to cis isomer which is equivalent to an increase of fraction of parallel (shorter) pairs. The calculated variation of $\Delta T_{\mathrm{NI}}$ with respect to incident $\mathrm{UV}$ radiation energy shows a qualitative agreement with experimental trends. The model is being further extended to include the smectic interaction and further work is under progress.

\section{References}

[1] P.G. de Gennes, J. Prost, The Physics of Liquid Crystals, 2nd ed, Clarendon Press, Oxford 1993.

[2] S. Chandrasekhar, Liquid Crystals, 2nd ed., Cambridge University Press, Cambridge 1992.

[3] C.H. Legge, G.R. Mitchell, J. Phys. D, Appl. Phys. 25, 492 (1992); (a) H.J. Coles, H.G. Walton, D. Guillon, G. Poetti, Liq. Cryst. 15, 551 (1993); (b) T. Ikeda, O. Tsutsumi, Science 268, 1873 (1995); (c) S. Servaty, F. Kremer, A. Schonfeld, R. Zentel, Z. Phys. Chem. (Leipzig) 190, 73 (1995); (d) G.G. Nair, S.K. Prasad, C.V. Yelamaggad, J. Appl. Phys. 87, 2084 (2000); (e) N. Tamaoki, Adv. Mater. 13, 1135 (2001); (f) V. Ajay Mallia, Mathew George, Suresh Das, Chem. Mater. 11, 207 (1999); (i) S. Krishna Prasad, Geetha G. Nair, K.L. Sandhya, D.S. Shankar Rao, Curr. Sci. 86, 815 (2004); T. Ikeda, J. Mat. Chem. 13, 2037 (2003).

[4] C. Yeh, Applied Photonics, Academic Press, San Diego 1994.

[5] E. Santamato, B. Daino, M. Romagnoli, M. Settembre, Y.R. Shen, Phys. Rev., Lett. 57, 2423 (1986).

[6] (a) I. Janossy, A.D. Lloyd, Mol. Cryst. Liq. Cryst. 203, 77 (1991); (b) T. Sasaki, K. Ichimura, Nature (London) 361, 428 (1993).

[7] (a) H. Knobloch, H. Orendi, M. Buchel, T. Seki, S. Ito, W. Knoll, J. Appl. Phys. 77, 481, (1995); (b) L.M. Blinov, M.V. Kozlovsky, M. Ozaki, K. Skarp, K. Yoshino, J. Appl. Phys. 84, 3860 (1998); L.M. Blinov, J. Nonlinear Opt. Phys. Mater. 5, 165, (1996); (c) M. Eich, B. Reck, H. Ringsdorf, J.H. Wendorff, Proc. SPIE. 682, 93 (1986); (d) B. Fischer, C. Thieme, T.M. Fischer, F. Kremer, T. Oge, R. Zentel, Liq. Cryst. 22, 65, (1997); (e) Y. Lansac, M.A. Glaser, N.A. Clark, O.D. Lavrentovich, Nature (London) 398, 54 (1999); T.A. Krentsel, O.D. Lavrentovich, S. Kumar, Mol. Cryst. Liq. Cryst. 304, 463 (1997). 
[8] W.E. Haas, K.F. Nelson, J.E. Adams, G.A. Dir, J. Electrochem. Soc. 121, 1667 (1974).

[9] D. Meyerhofer, J. Appl. Phys. 46, 5084 (1975).

[10] S. Krishna Prasad, Geetha G. Nair, Gurumurthy Hegde, K.L. Sandhya, D.S. Shankar Rao, Chethan V. Lobo, C.V. Elamaggad, Phase Transit. 78, 443, (2005).

[11] Gurumurthy Hegde, Geetha G. Nair, S. Krishna Prasad, C.V. Yelamaggad, J. Appl. Phys. 97, 093105 (2005).

[12] N.V. Madhusudana, Jyothsna Rajan, Liq. Cryst. 7, 31 (1990)

[13] G. Basappa, N.V. Madhusudana, Eur. Phys. J. B. 1, 179 (1998).

[14] N.V. Madhusudana, S. Chandrasekhar, Pramana Suppl. 1, 57 (1973).
[15] A.S. Govind, Ph.D. Thesis, Raman Research Institute, Bangalore 2002, Ch. 2, p. 38.

[16] (a) A.S. Govind, N.V. Madhusudana, Liq. Cryst. 23 327 (1997); (b) A.S. Govind, N.V. Madhusudana, Liq. Cryst. 27, 215 (2000).

[17] A.S. Govind, N.V. Madhusudana, Liq. Cryst. 14, 1539 (1993)

[18] S. Krishna Prasad, Geetha G. Nair, K.L. Sandhya, D.S. Shankar Rao, Current Science 86, 815 (2004).

[19] Geetha Basappa, A.S. Govind, N.V. Madhusudana, J. Phys. II (France) 7, 1693 (1997). 